Document downloaded from:

http://hdl.handle.net/10251/94493

This paper must be cited as:

Payri, R.; F.J. Salvador; Gimeno, J.; García Martínez, A. (2012). Flow regime effects over non-cavitating diesel injection nozzles. Proceedings of the Institution of Mechanical Engineers Part D Journal of Automobile Engineering. 226(1):133-144. doi:10.1177/0954407011413056

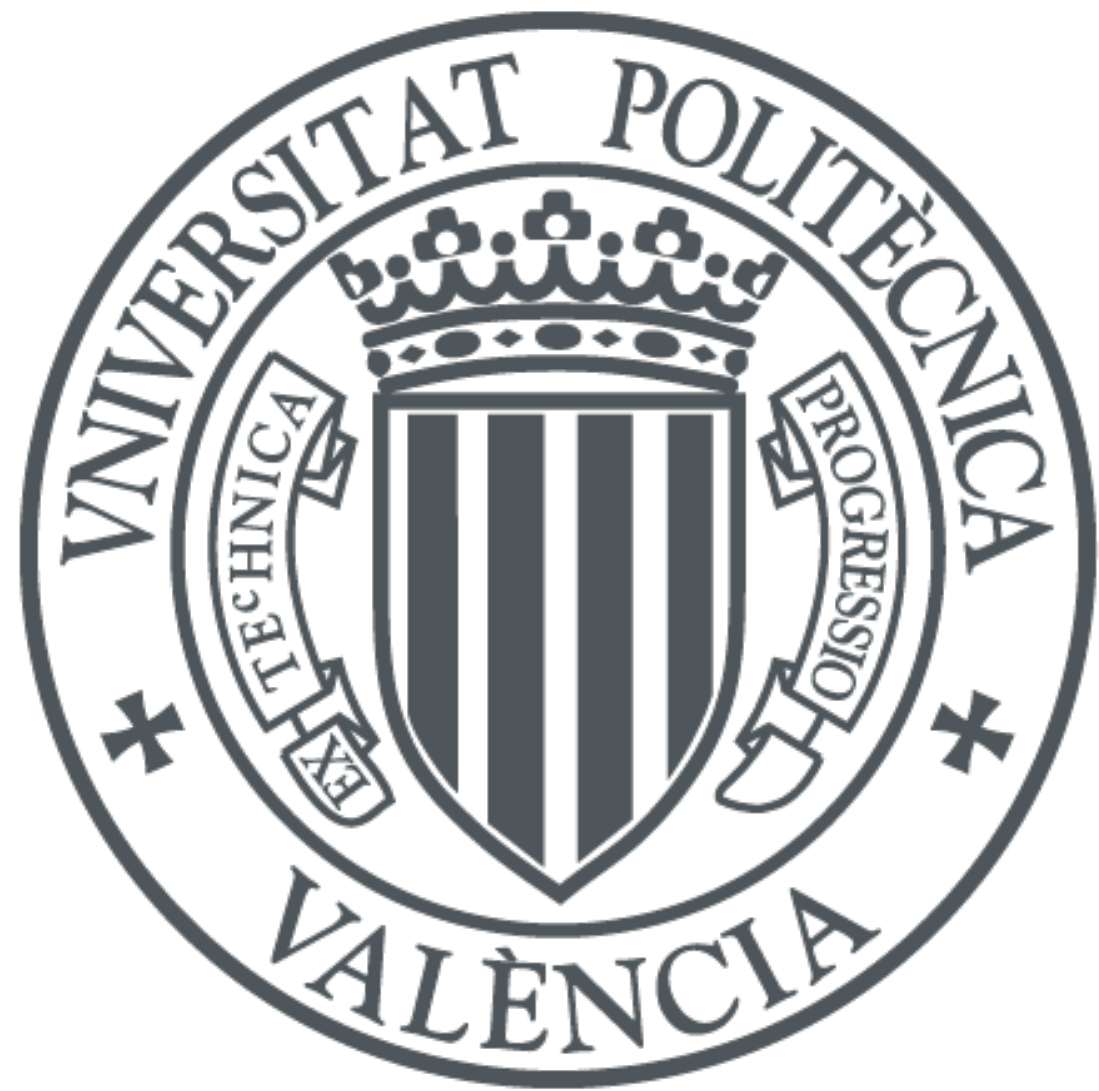

The final publication is available at

http://doi.org/10.1177/0954407011413056

Copyright SAGE PUBLICATIONS LTD

Additional Information 


\title{
Flow regime effects over non-cavitating diesel injection nozzles
}

\author{
R. Payri* F.J. Salvador, J. Gimeno, A. García \\ CMT-Motores Térmicos, Universidad Politécnica de Valencia, \\ Camino de Vera s/n, Valencia E-46022, Spain
}

May 17, 2011

\begin{abstract}
The research conducted and explained in this paper aims exploring and understanding the influence of flow regime (laminar, transition or turbulent) inside diesel injector nozzles. For this purpose, an experimental study based on mass flow rate and momentum flux measurements on three convergent nozzles has been carried out. The combination of both types of measurements has been helpful to obtain information about the nature of the flow and its consequences on important variables, such as injection effective velocity and effective area of nozzle outlet orifices. As a main result of the investigation, and depending of the flow regime, a differentiated behavior has been observed which was clearly reflected in the non dimensional flow parameters defined and used through the study.
\end{abstract}

\section{Nomenclature}

$A$ Area.

$A_{e f} \quad$ Effective area.

$A_{o} \quad$ Orifice outlet section area.

$A R$ Orifice area reduction.

$C_{a}$ Area Coefficient.

$C_{d}$ Discharge coefficient.

$C_{M}$ Momentum coefficient.

${ }^{*}$ Corresponding author. Email: rpayri@mot.upv.es 
$C_{v}$ Velocity coefficient.

$C_{d u}$ Ultimate discharge coefficient.

$D_{i}$ Inlet diameter.

$D_{o}$ Outlet diameter.

$D_{e f} \quad$ Effective diameter.

$f$ Friction factor.

$k$-factor Orifice conicity factor.

$L$ Orifice length.

$L_{i} \quad$ Inlet length until developed flow.

$\dot{M}$ Momentum flux in axial direction.

$\dot{m}$ Mass flux.

$p_{b} \quad$ Back pressure.

$p_{i}$ Injection pressure.

$r$ Inlet curvature radius.

Re Reynolds number.

$u$ Velocity in axial direction.

$u_{m}$ Mean velocity in a tube.

$u_{e f}$ Effective velocity.

$u_{\text {berno }}$ Maximal theoretical Bernoulli velocity.

$\Delta p \quad$ Pressure drop across the nozzle.

$\Delta p_{\text {inlet }}$ Pressure losses at inlet tube.

$\Delta p_{\text {fric }} \quad$ Wall friction pressure losses.

$\delta$ Boundary layer thickness

$\gamma$ Intermittency factor.

$\nu$ Kinematic viscosity.

$\rho_{f}$ Fuel density.

$\xi$ Pressure losses coefficient.

\section{Introduction}

In the last years, the injection system research has raised a great interest in the diesel engine framework. Considering a mixing controlled combustion, the air-fuel mixing process governs mainly the engine performance and pollutant emissions, therefore, the above mentioned interest is not accidental, due to the quality of the air-fuel mixture will largely depend on the proper work of the injection system $[1,2,3,4]$.

Before improving the injection system performance is necessary to understand deeply all the phenomena involved. Undoubtedly, one of the most critical elements on this process is the injection nozzle and, more specifi- 
cally, the geometry of the discharge outlets. This geometry will influence the characteristics of internal flow and spray development $[5,6,4,7]$.

However, despite of the influence of the flow characteristics inside the injection orifices on the injection and combustion processes, the physics of this flow is currently unknown. This lack of knowledge is partly justified by the enormous difficulty of studying the internal flow: the small size of the orifices, the high flow velocities and the strongly transient flow. These features make the experimental study of the flow inside the orifices of the injection nozzles quite complicated [4].

Improvements in nozzle manufacturing techniques have made possible to perform convergent orifices with the aim of preventing cavitation [8], besides of improving the discharge coefficient. In recent years, engine manufacturers have chosen precisely these convergent nozzles to the detriment of the cylindrical and cavitating ones. Convergent nozzles offer more consistent and predictable behavior independently of pressure conditions used in the engine operating map. These trends are not extended with cylindrical nozzles where different pressure can lead to cavitation at some points and not cavitation in other ones, generating diverse situations depending on the cavitation appearance and leading to a flow behavior at the orifice outlet completely different with an effective area much smaller in the case of cavitating conditions [9].

As a first approach and because of the high velocities and high Reynolds numbers inside injection nozzles, in diesel injection conditions, it is reasonable to think that the flow could be always in turbulent conditions even for low injection pressures. However, as discussed in this work, this hypothesis is not always correct, and can exist points at low injection pressure conditions where the flow is not in turbulent conditions and therefore affecting both internal flow characteristics and the subsequent spray development. This way, this fact must be taken in consideration when injection nozzle is designed or injection mapping is set up for a given engine, even more taking into account that diesel injection nozzles are getting smaller and more convergent.

This work is focused on revealing the effects of the flow regime on the nozzles internal flow. For this purpose, an experimental study using three convergent nozzles with different diameters and different pressure conditions, on which is measured not only the mass flow but also the momentum flux. The great interest of both measurements, mass and momentum flux, is the possibility of estimating parameters such as velocity and effective area at the nozzle outlet and the dimensionless coefficients [9]. Using these parameters, on the one hand, it can be determined which is the flow regime at each tested point, and on the other hand, it is possible to determine at each case the effect of regime type on flow characteristics. Moreover, the study of three 
different nozzles allows determining the influence of the orifice diameter size, or the influence of the length-diameter ratio, on the regime and the internal flow behavior.

So, the current paper is divided into three parts. In the first part, theoretical aspects of flow are discussed, with the definition of different flow parameters used (Section 2). In the second part the experimental techniques employed are briefly presented (Section 3), and the nozzles and operating conditions tested (Section 4). And in the last part, the obtained results are presented (Section 5), and the analysis is performed (Section 6).

\section{Internal flow parameters and coefficients}

\subsection{Mass and momentum flux}

The mass flux thought the outlet section of an injection orifice, see Figure 1a, can be obtained as

$$
\dot{m}=\int_{A_{o}} \rho u d A,
$$

where $A_{o}$ is the geometric section area, $u$ is the axial component of velocity, and $\rho$ is the density profile.

In the same way, the momentum flux thought the outlet section of an injection orifice, and considering only the axial component, can be obtained as

$$
\dot{M}=\int_{A_{o}} \rho u^{2} d A,
$$

being $\dot{M}$ the axial component of the momentum flux at orifice outlet.

\subsection{Effective velocity and area}

From the mass and momentum flux measurements is possible to determine two useful internal flow parameters: effective velocity and effective area at orifice outlet.

The real flow at the orifice outlet will be determined by the profiles of velocity and density, Figure 1a. In this flow, it is possible to measure, and therefore to know, both momentum and mass flux, as the integral, according to equations (1) and (2).

The characteristics of these profiles are totally unknown. However, it is possible to define an effective velocity and area representatives of the flow. The definition of these parameters is based on a simplified flow, which is 


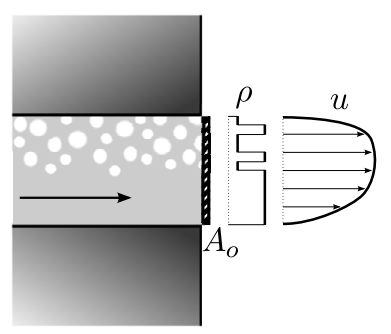

$\mathbf{a}$

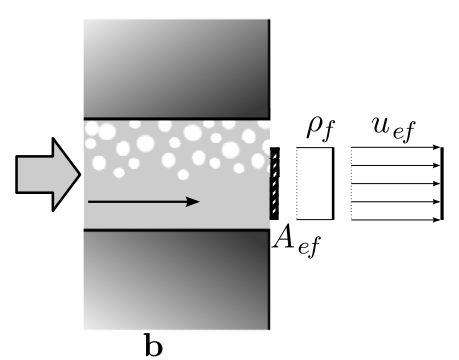

b

Figure 1: Effective velocity and area definition.

characterized by an effective area $A_{e f}$, lower than the geometric one, through the flow goes out with an uniform effective velocity $u_{e f}$, and with a density equal to the liquid one $\rho_{f}$, see Figure 1b. Moreover, the value of these parameters, $u_{e f}$ and $A_{e f}$, must be such that the mass and momentum flux for the simplified flow (Figure 1b) will be equal to the real situation (Figure 1a).

Solving the integral equations of mass and momentum flux ((1) and (2)) for the simplified case it is obtained

$$
\begin{gathered}
\dot{m}=A_{e f} \rho_{f} u_{e f} \\
\dot{M}=A_{e f} \rho_{f} u_{e f}^{2}
\end{gathered}
$$

Now, from these two equations is possible to obtain the effective velocity and area, as function of the mass and momentum flux, which are known as

$$
\begin{gathered}
u_{e f}=\frac{\dot{M}}{\dot{m}} \\
A_{e f}=\frac{\dot{m}^{2}}{\rho_{f} \dot{M}}
\end{gathered}
$$

Moreover, if a circular area is supposed, it can be obtained the effective diameter as

$$
D_{e f}=\sqrt{\frac{4 A_{e f}}{\pi}} .
$$

\subsection{Maximum theoretical Bernoulli velocity}

The Bernoulli velocity represents the maximum theoretical velocity that can be achieved in the nozzle orifice outlet, for a given pressure drop. In order to evaluate it, the Bernoulli equation between upstream and downstream of 
the orifice is used. Thus, assuming negligible upstream velocity and no losses between upstream and downstream, it is obtained

$$
\frac{p_{i}}{\rho_{f}}=\frac{p_{b}}{\rho_{f}}+\frac{1}{2} u_{b e r n o}^{2}
$$

where $p_{i}$ and $p_{b}$ are upstream or injection pressure and downstream or back pressure respectively, and $\rho_{f}$ is the fuel density. Isolating from this equation, and taking into account that the pressure drop at the orifice is $\Delta p=p_{i}-p_{b}$, the maximum theoretical velocity, also known as Bernoulli velocity, is defined as

$$
u_{\text {berno }}=\sqrt{\frac{2 \Delta p}{\rho_{f}}} .
$$

\subsection{Flow coefficients}

In this section, the dimensionless coefficients of the above parameters: mass flux, momentum flux, effective velocity and effective area, will be defined:

Velocity coefficient. This coefficient relates the effective velocity with the maximum theoretical Bernoulli velocity (equation (9)). It is calculated with the following equation

$$
C_{v}=\frac{u_{e f}}{u_{\text {berno }}}=\frac{u_{e f}}{\sqrt{2 \Delta p / \rho_{f}}} .
$$

Area coefficient. This coefficient is used for evaluating the reduction of the effective area regarding to geometric one, and it is calculated as

$$
C_{a}=\frac{A_{e f}}{A_{o}} .
$$

Discharge coefficient. This coefficient is defined as the real measured mass flux regarding to the maximum theoretical mass flux[10]. The maximum mass flux is evaluated considering uniform velocity and equal to Bernoulli velocity and using all the geometric section area.

$$
C_{d}=\frac{\dot{m}}{\dot{m}_{\text {th }}}=\frac{\dot{m}}{A_{o} \rho_{f} u_{\text {berno }}}=\frac{\dot{m}}{A_{o} \sqrt{2 \rho_{f} \Delta p}} .
$$


Momentum coefficient. As in the mass flux, the momentum coefficient is defined as the real measured momentum flux regarding to the maximum theoretical momentum flux. Where the maximum momentum flux is evaluated considering the Bernoulli velocity and the geometric area.

$$
C_{M}=\frac{\dot{M}}{\dot{M}_{\text {th }}}=\frac{\dot{M}}{A_{o} \rho_{f} u_{\text {berno }}^{2}}=\frac{\dot{M}}{2 A_{o} \Delta p} .
$$

\subsubsection{Relations between coefficients}

If in the $C_{d}$ coefficient, equation (12), mass flux is replaced with effective parameters, it is obtained

$$
C_{d}=\frac{A_{e f} \rho_{f} u_{e f}}{A_{o} \rho_{f} u_{\text {berno }}} .
$$

Combining this equation with equations (11) and (10), it is obtained

$$
C_{d}=C_{a} C_{v}
$$

Operating as above the momentum coefficient it is obtained

$$
C_{M}=C_{a} C_{v}^{2} .
$$

Finally, combining this two relations

$$
C_{v}=\frac{C_{M}}{C_{d}} .
$$

\subsection{Flow parameters interpretation}

The area coefficient, $C_{a}$, is used to evaluate the losses of effective section area with regard to the geometric area. The lower the $C_{a}$, the bigger area losses. The losses of effective area are due to the following possible causes:

- Non-uniform velocity profile at orifice outlet.

- Cavitation cavities reaching the orifice outlet.

- Flow separation from the wall (hydraulic-flip).

Considering these causes, it is expected that a full developed turbulent flow without cavitation will have $C_{a} \approx 1$ due to its uniform velocity profile. And contrarily, lower $C_{a}$ will be obtained when cavitation or flow separations appears due to the decrease of density. 
The velocity coefficient, $C_{v}$, compares the effective velocity with the Bernoulli velocity. The Bernoulli velocity is the velocity that will be achieved if all the pressure energy is transformed in kinetic energy without losses. So, this parameter is useful in order to evaluate the pressure or energy losses that occurs in the injection process. These losses will be concentrated mainly in the injection orifices, since it is here where the flow finds the principal section restriction, so the $C_{v}$ value will depend mainly on the nozzle orifices geometry. Although it is important to remember that this coefficient takes into account all the energy losses that take place from the upstream (where the injection pressure is measured) to the downstream (the orifice outlet). So, special consideration must be taken in some situations, for example, when different types of injectors are compared.

\section{Experimental facilities}

In order to perform this study, a commercial common rail and two experimental set-ups have been used together with a methodology for obtaining the internal geometry of diesel nozzles. A brief description of these tools will be made in this section.

\subsection{Internal geometry determination}

A special type of silicone have been introduced inside the nozzles, as described by Macián et al. [11] in order to analyze the internal characteristics of the nozzles used in the current investigation. The silicone moulds have been visualized in a microscope where several pictures of the most relevant geometrical parameters have been taken. An example of the pictures taken for one of the nozzles used in this study is presented in Figure 2

\subsubsection{Geometry nomenclature}

In Figure 3 is defined the most significant dimensions of an injection orifice: Inlet and outlet diameters $D_{i}$ and $D_{o}$, length $L$, and inlet radius $r$. From these dimensions are defined the following dimensionless parameters of the orifices:

- Length-diameter ratio $L / D=L / D_{o}$.

- Inlet curvature radius-diameter ratio $r / D=r / D_{i}$. This parameter determines the design of the inlet orifice. For sharp edges this value will be zero. 

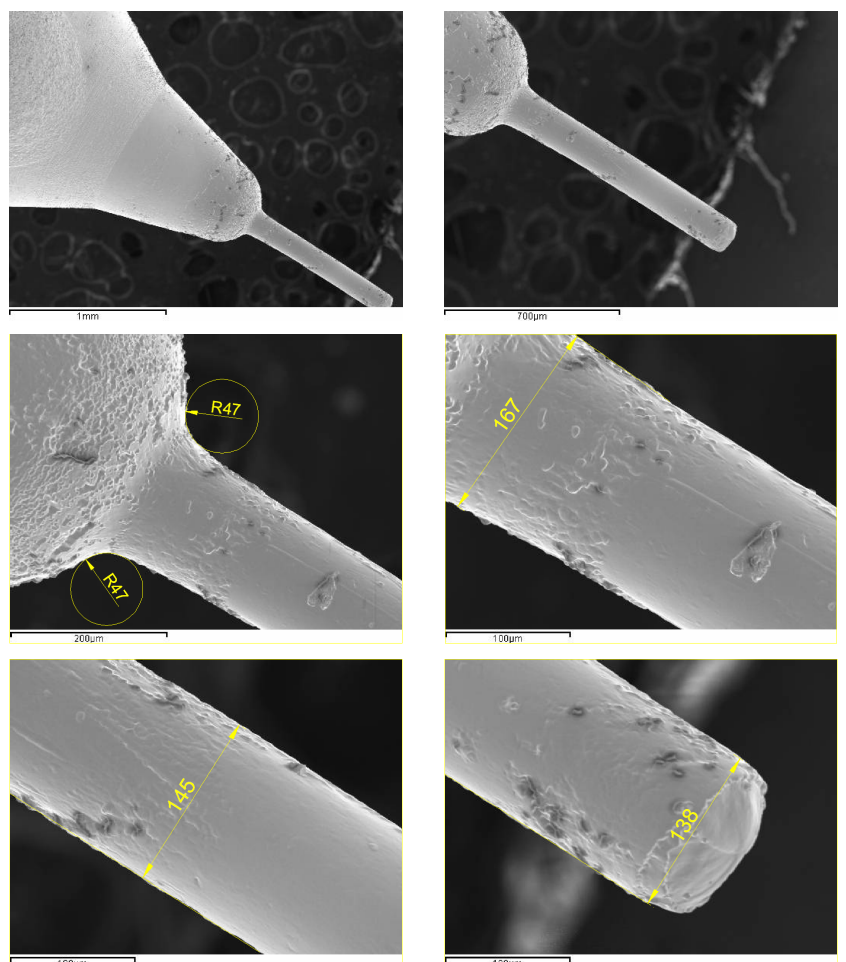

Figure 2: Processed images of silicone for nozzle B.

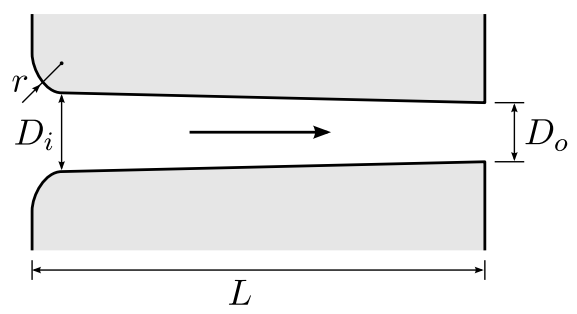

Figure 3: Geometric parameters of a injection orifice. 
- Inlet and outlet diameters ratio $D_{i} / D_{o}$. This parameter determines the orifice convergence or divergence. This parameter will be the unity for cylindrical orifices and for convergent orifices is greater than one.

To determine the orifice convergence in the literature is common the use of $k$-factor parameter which is calculated as:

$$
k-\text { factor }=\frac{D_{i}-D_{o}}{10[\mu \mathrm{m}]} .
$$

However, this parameter has a significant weakness, because does not distinguish between different sizes of orifices. For example, an orifice with $D_{i}=210 \mu \mathrm{m}$ and $D_{o}=200 \mu \mathrm{m}$ has the same $k$-factor than another of $D_{i}=110 \mu \mathrm{m}$ and $D_{o}=100 \mu \mathrm{m}$, however, the area variation, and therefore the flow characteristics, in each case are completely different. A most appropriate parameter to evaluate the orifice convergence is the area reduction, defined as [11].

$$
A R=\frac{A_{i}-A_{o}}{A_{i}}=\frac{D_{i}^{2}-D_{o}^{2}}{D_{i}^{2}}
$$

\subsection{Injection Rate Meter}

Measurements of injection rate were carried out with an Injection Discharge Rate Curve Indicator (IRDCI) commercial system. The device makes it possible to display and record the data that describe the chronological sequence of an individual fuel injection event. The measuring principle used is the Bosch method [12], which consists of a fuel injector that injects into a fuelfilled measuring tube. The fuel discharge produces a pressure increase inside the tube, which is proportional to the increase in fuel mass. The rate of this pressure increase corresponds to the injection rate. A pressure sensor detects this pressure increase, and an acquisition and display system further processes the recorded data for further use. Mass flux signals has been processed using the methodology described in [13].

\subsection{Spray Momentum test Rig}

With this experimental equipment it is possible to determine the impact force of a spray on a surface. This force is equivalent to the spray momentum flux. Figure 4 shows a sketch of the momentum test rig. Sprays are injected into a chamber that can be pressurized with nitrogen up to $10 \mathrm{MPa}$, in order to simulate pressure discharge conditions that are representative of 
real pressure conditions inside the engine combustion chamber during the injection process.

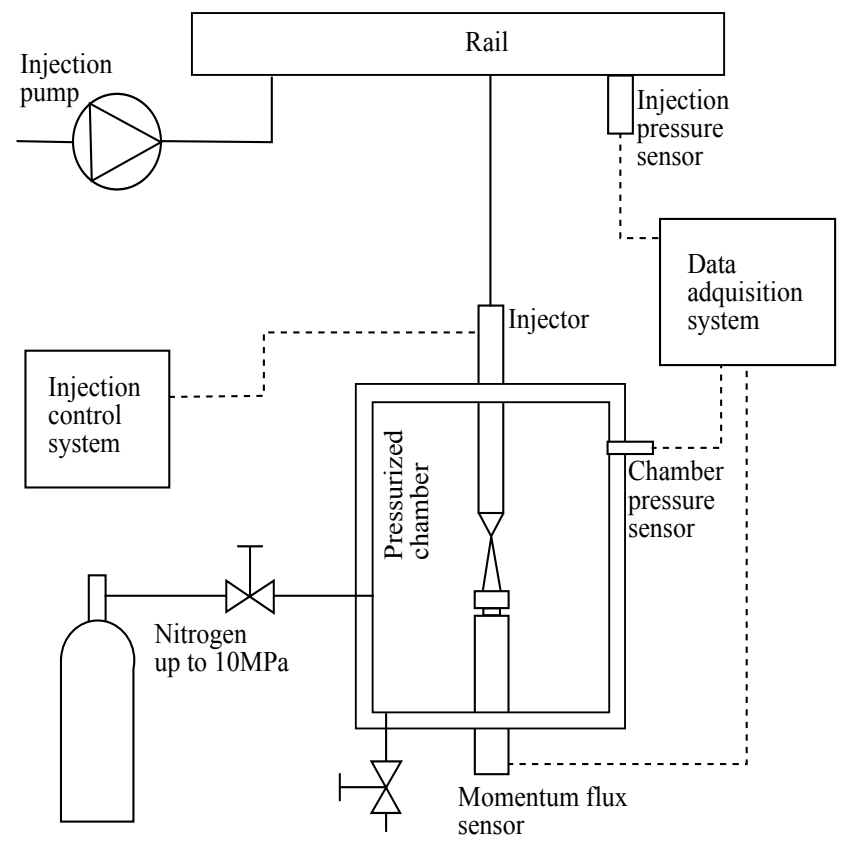

Figure 4: Spray momentum test rig.

Figure 5 shows a sketch of the spray momentum measuring principle. The impact force is measured with a calibrated piezo-electric pressure sensor in order to measure force. The sensor is placed at $5 \mathrm{~mm}$ from the hole exit and its target has $8 \mathrm{~mm}$ of diameter. The sensor frontal area and position are selected so that spray impingement area is much smaller than that of the sensor. Under this assumption, and due to the conservation of momentum, the force measured by the sensor will be the same as the momentum flux at the hole outlet or at any other axial location, since the pressure inside the chamber is constant and surrounds the entire spray and fuel deflected is perpendicular to the axis direction.

\section{Studied nozzles and operating conditions}

To perform this study it has been chosen three different one hole axi-symmetric nozzles. As it has been mentioned earlier, the orifices are convergent with conical shape, additionally; the nozzles have been manufactured with an ag- 


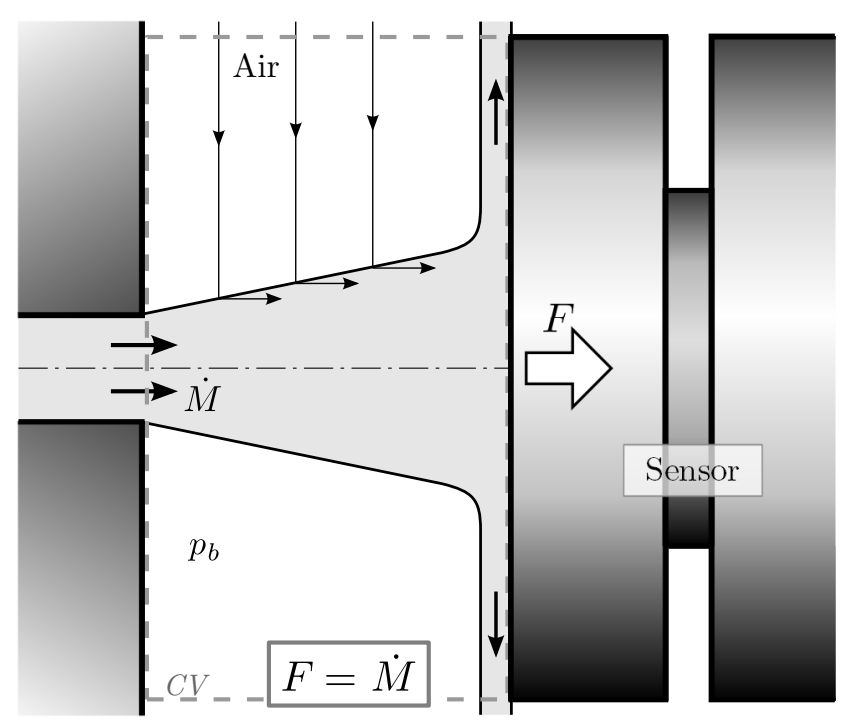

Figure 5: Spray momentum measurement principle.

gressive hydroerosion process to round the edges of orifice inlet providing them an inlet radius large enough to avoid separation of the boundary layer at the orifice inlet. Both characteristics, convergent orifices and large inlet radius, will prevent the cavitation phenomenon[14]. Besides, it can be expected that these types of orifices have few losses enabling a large discharge capacity.

On these nozzles has been used the mold silicone technique [11] with the aim of obtaining the internal geometry of orifices. Results for three nozzles are summarized in Table 1, while the characteristic of nozzles dimensionless geometric parameters are shown in Table 2. Unfortunately it has not been possible to obtain the complete mould for nozzle $\mathrm{C}$, so $D_{o}$ value used in the current research is the supplied by nozzle manufacturer.

Table 1: Internal geometry of the studied one-hole nozzles.

\begin{tabular}{lcccccc}
\hline Nozzle & $r$ & $D_{i}$ & $D_{m}$ & $D_{o}$ & $k$-factor & $A R$ \\
& $\mu \mathrm{m}$ & $\mu \mathrm{m}$ & $\mu \mathrm{m}$ & $\mu \mathrm{m}$ & - & $\%$ \\
\hline $\mathrm{A}$ & 42 & 140 & 125 & 112 & 2.8 & 36.0 \\
$\mathrm{~B}$ & 47 & 167 & 145 & 138 & 2.9 & 31.7 \\
$\mathrm{C}$ & 49 & 195 & & 156 & 3.9 & 36.0 \\
\hline
\end{tabular}

As it is shown in these tables, the three nozzles are similar. The unique 
Table 2: Dimensionless parameters of the studied one-hole nozzles.

\begin{tabular}{lcccc}
\hline Nozzle & $D_{o}$ & $L / D$ & $r / D$ & $D_{i} / D_{o}$ \\
\hline $\mathrm{A}$ & $112 \mu \mathrm{m}$ & 8.93 & 0.30 & 1.25 \\
$\mathrm{~B}$ & $138 \mu \mathrm{m}$ & 7.25 & 0.28 & 1.21 \\
$\mathrm{C}$ & $156 \mu \mathrm{m}$ & 6.41 & 0.25 & 1.25 \\
\hline
\end{tabular}

difference between them is the orifice diameter. It is noteworthy the strong orifices convergence and the great inlet radius.

\subsection{Operating conditions}

In the current research the following test plan with four different injection pressures and four different discharge pressures is used. It has been selected a long energizing time of $2000 \mu \mathrm{s}$, with the aim of achieving stationary values of the injection event when the needle lift gets its maximum position.

$$
\begin{array}{ll}
\text { Injection pressure } & 30-80-130-150 \mathrm{MPa} \\
\text { Discharge pressure } & 2.5-3.5-5-8 \mathrm{MPa} \\
\text { Energizing time } & 2000 \mu \mathrm{s}
\end{array}
$$

In both experimental set-ups injection pressure has been measured by means of a piezoresistive high pressure sensor located at common rail. Fortunately, since the mass flow of these one-hole nozzles is small (compared to conventional multi-hole ones), pressure loss between the pressure measurement point and the orifice inlet will be also small.

\subsection{Fuel characteristics}

A Repsol CEC RF-06-99 diesel fuel, with a density of $820.2 \mathrm{~kg} / \mathrm{m} 3$ and a kinematic viscosity of $2.67 \mathrm{~mm} 2 / \mathrm{s}$ (measured at $40^{\circ} \mathrm{C}$ ), was used as fuel in the experiments. The summary of some physical and chemical properties is shown in Table 3.

Table 3: Physical properties of Repsol CEC RF-06-99 fuel.

\begin{tabular}{lccc}
\hline Test & Unit & Result & Uncertainty \\
\hline Density at $15^{\circ} \mathrm{C}$ & $\mathrm{kg} / \mathrm{m}^{3}$ & 843 & 0.2 \\
Viscosity at $40^{\circ} \mathrm{C}$ & $\mathrm{mm}^{2} / \mathrm{s}$ & 2.847 & 0.42 \\
\hline
\end{tabular}




\section{Results}

In Figures 6 and 7 are shown some examples of signals obtained for the momentum flux and the injection rate respectively. In these figures can been appreciated as larger the orifice diameter, higher is the momentum flux and mass flow.

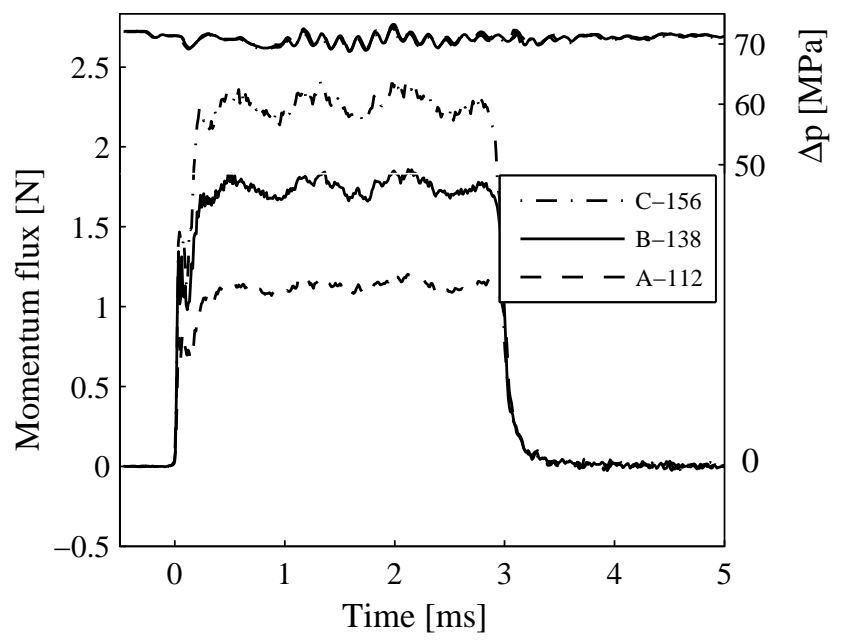

Figure 6: Temporal evolution of the momentum flux. $p_{i}=80 \mathrm{MPa} p_{b}=$ $3.5 \mathrm{MPa}$.

Through the large energizing time used, the signals have a large area in which the needle is at maximum lift. To perform the following analysis is considered only this stationary area, from which the momentum flux average values, the mass flow and its respective injection pressures in each point tested are extracted. Therefore, with the aim of avoiding the effect that may cause the needle lift, the initial and final period of the test has been neglected.

In the following section the results for the mass flow, the momentum flux, the velocity, the effective area and the different coefficients are calculated.

The uncertainty in the determination of the coefficients were estimated using standard procedure, as outlined by Holman [15]. The uncertainty obtained for the discharge coefficient was around $1.5 \%$ and $1.8 \%$ for the momentum coefficient. 


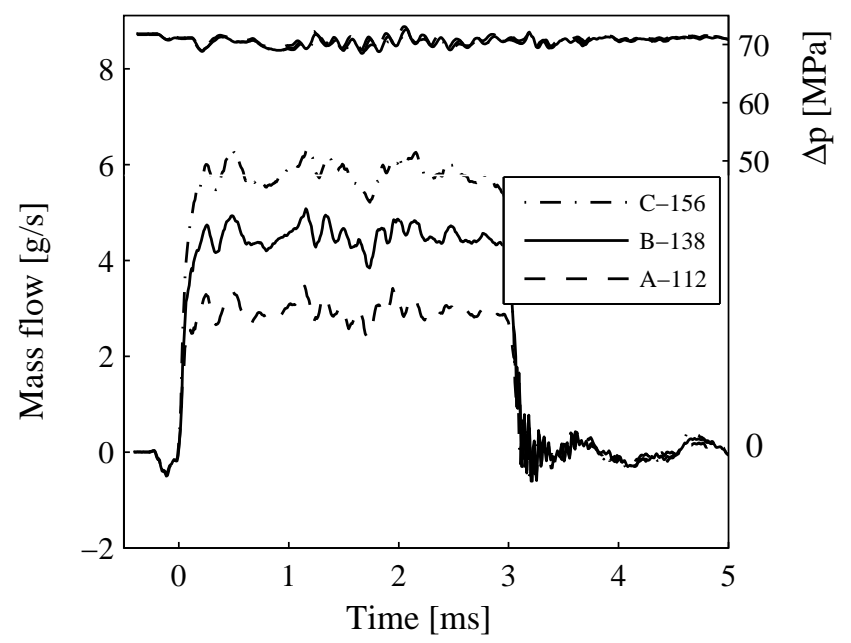

Figure 7: Temporal evolution of the injection rate. $p_{i}=80 \mathrm{MPa} p_{b}=$ 3.5 MPa.

\subsection{Mass flow}

In Figure 8 is represented the mass flow rate versus square root of pressure drop. Each group of points corresponds to an injection pressure. The gray level distinguishes the different back-pressures being darker for higher values. The mass flow behavior is linear with the square root of the pressure drop as is expected for no cavitant nozzles. In addition, it can also be observed that as the larger is the diameter the higher is the mass flow.

The dimensionless of these data can be performed calculating the discharge coefficient, $C_{d}$, which represents the relation between the real mass flow compared to the theoretical calculated with pressures involved. In Figure 9 is shown the discharge coefficient against the Reynolds number calculated from Bernoulli theoretical velocity $R e=u_{\text {berno }} D_{o} / \nu$.

In this figure, a similar behavior for the different nozzles is observed, nevertheless, in each of them can be distinguished two zones clearly:

- The first corresponds to low Reynolds numbers (Re $\lesssim 15000)$, in this zone $C_{d}$ has a growing trend. It is worthy to note how the three nozzles, in spite of being different, collapse in the same zone for the same Reynolds number. This behavior is slightly strange because the nozzles have different relationship $L / D$, however, they behave similarly [16], so this behavior could be simply circumstantial. 


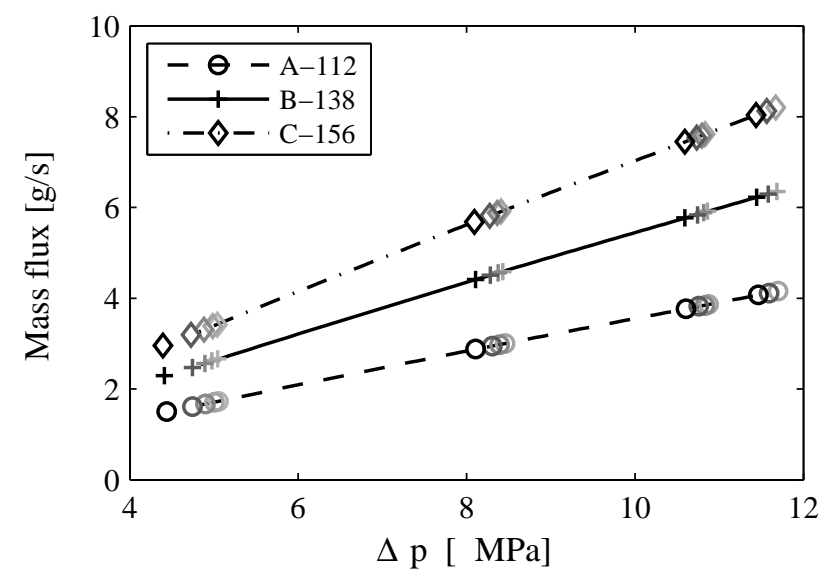

Figure 8: Mass flow rate versus square root of pressure drop.

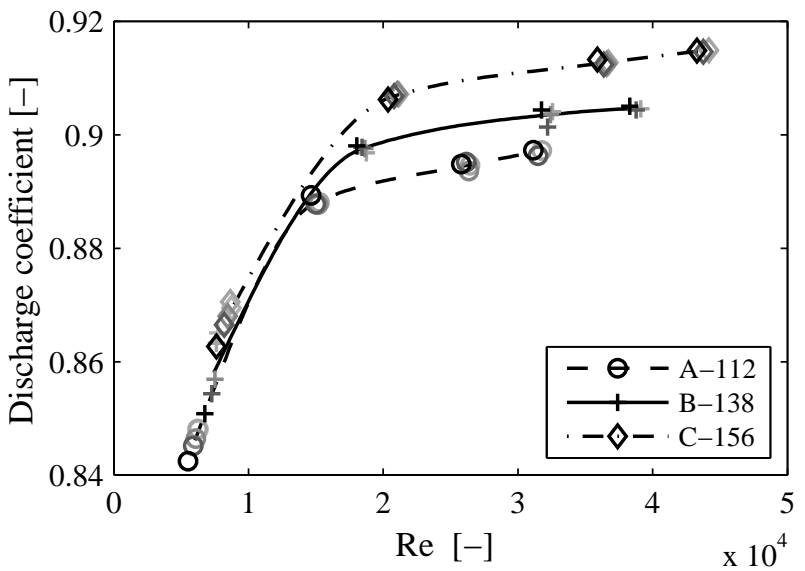

Figure 9: Discharge coefficient versus Reynolds number. 
- At high Reynolds numbers, the $C_{d}$ reduces its growth tendency showing more stable behavior except for a slight increase with increasing $R e$. The $C_{d}$ stabilized value is known as maximum or last, $C_{d u}$ [17]. This maximum value is different for each nozzle, being greater when the diameter is higher and therefore the ratio $L / D$ is lower.

This behavior has been observed on numerous occasions in non-cavitanting conditions $[17,18,19,20]$. The trend change is due to the transition of laminar regime (in growing zone) to the turbulent regime (in constant zone). The explanation of this behavior will be discussed later, with more information available on the internal flow.

The differences in the $C_{d u}$ are consistent with the results presented by Lichtarowicz [17] in orifices without cavitation. This author found the $C_{d u}$ decreases if the ratio $L / D$ increases. This behavior is due to higher friction losses with the walls if the orifice is longer, higher $L / D$.

\subsection{Momentum flux}

In Figure 10 the average values of momentum flux is presented . As it is expected, the momentum flux behaves proportional to the pressure drop. Additionally, the larger diameter, the higher momentum flux.

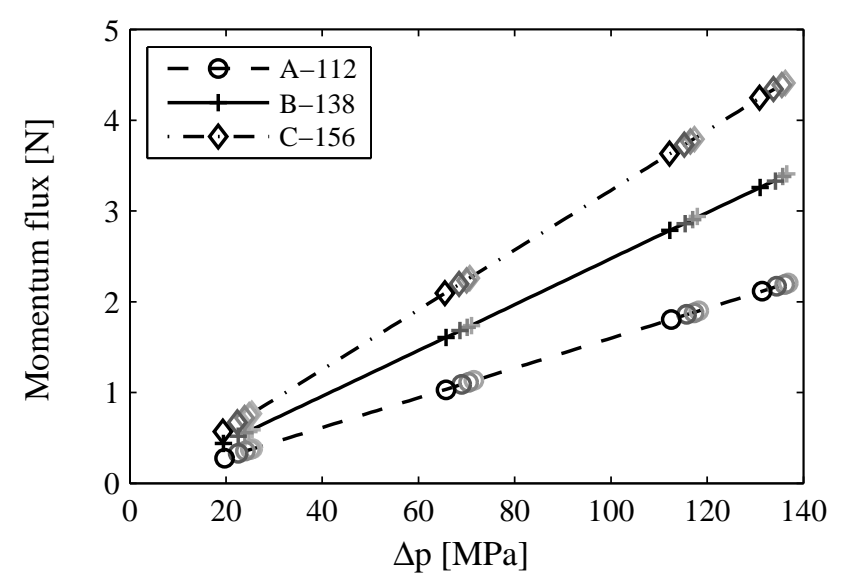

Figure 10: Momentum flux versus pressure drop.

As in mass flow case, these data can be converted to non dimensional dividing the real momentum flux by the theoretical; thereby the momentum coefficient $C_{M}$ or moment flux is obtained. In Figure 11 is presented the 
momentum coefficient respect to the Reynolds number. As it can be observed, the behavior of this coefficient is similar to the discharge coefficient. Although in this case is more difficult to distinguish between the two zones since there is no abrupt change in the coefficient trend.

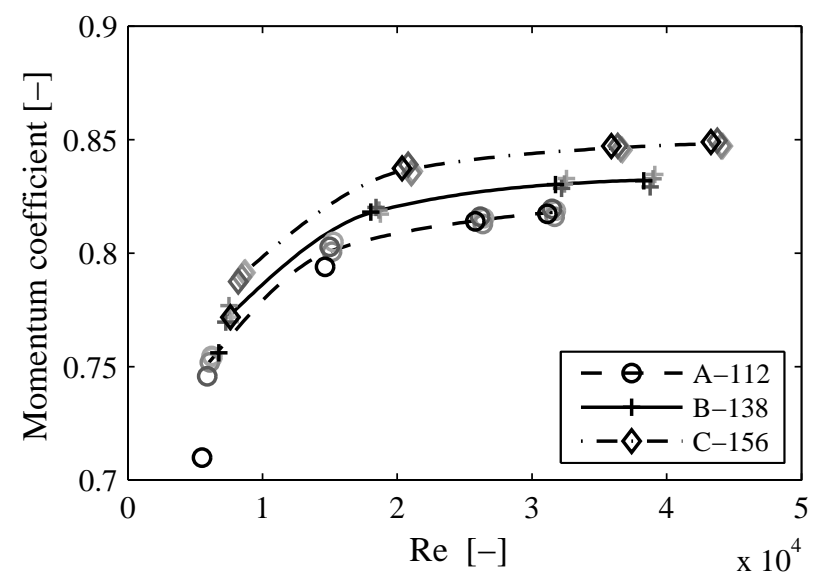

Figure 11: Momentum flux coefficient versus Reynolds number.

As it happened with the $C_{d}$ coefficient, the $C_{M}$ is greater when orifice diameter increases (decreases $L / D$ ). In this case, this behavior is reflected in both low and high Reynolds numbers.

\subsection{Effective velocity}

In this section the results for the effective velocity and the velocity coefficient calculated from the mass flow and momentum flux data are presented.

Figure 12 shows the outlet effective velocity versus the square root of the pressure drop. As it can be observed, actually, the effective velocity of all nozzles is very similar in all cases. This result is quite reasonable considering the velocity depends mainly on the pressure drop. The diameter, or more generally the orifice geometry, will not have a significant effect on the effective velocity, except for small differences that can exist in the velocity coefficient.

Despite being very similar the velocity obtained from the three nozzles, it can be noted, keeping constant the pressure drop, that a greater diameter means a slightly velocity increase. This effect is observed more clearly in the velocity coefficient represented in figure 13 . The explanation for this effect is related to an increase in friction losses with the walls when the $L / D$ ratio increase. 


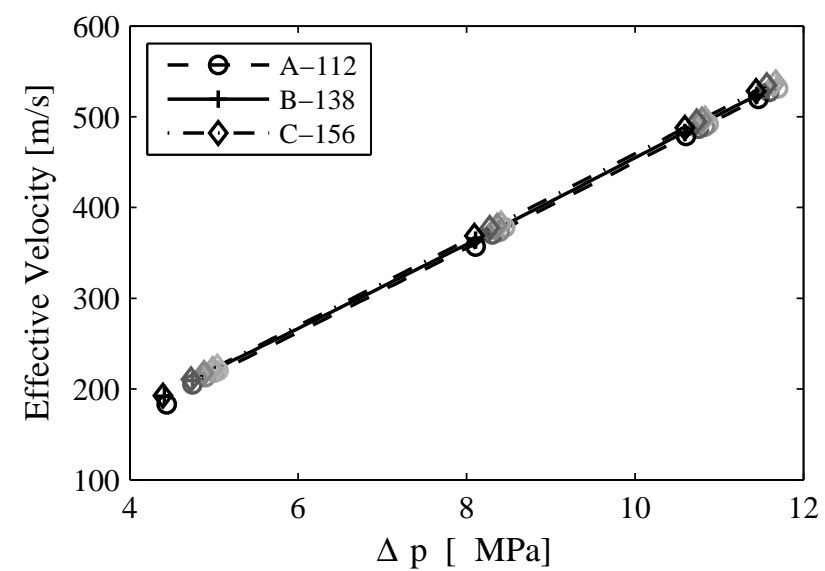

Figure 12: Effective velocity versus pressure increase.

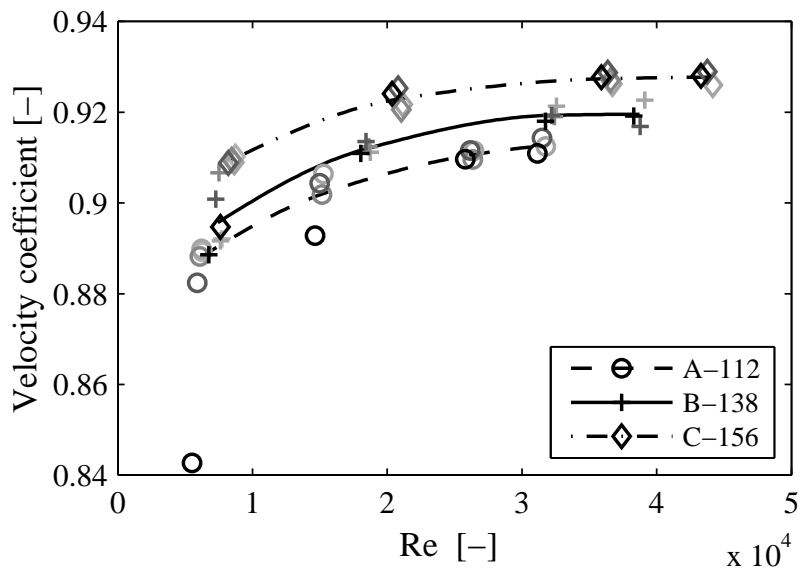

Figure 13: Velocity coefficient versus Reynolds number. 
The behavior of the velocity coefficient is very similar in the three nozzles, following an asymptotic trend in the three nozzles. However, unlike the behavior of the previous coefficients, in the case of velocity coefficient is impossible to distinguish between the two zones named above, showing a similar trend for low and high Reynolds numbers.

\subsection{Effective area}

In Figure 14 is represented the area coefficient calculated from the effective diameter and geometric diameter in function of Reynolds number.

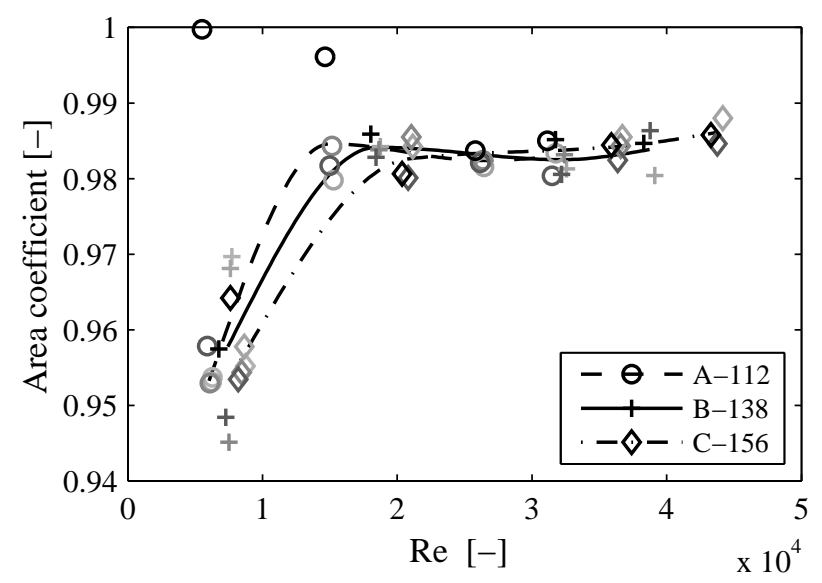

Figure 14: Area coefficient versus Reynolds number.

It can be observed the same behavior separated into two zones for all nozzles used in the present research: with low $R e$, the $C_{a}$ grows with the increase of Reynolds number to a maximum value close to one, from which, the $C_{a}$ remains constant forming the second zone. It is worthy to note how for the three nozzles a very similar stabilized value is achieved. These two zones are related to the behavior observed in the discharge coefficient.

The two zones appearance in $C_{a}$ coefficient contrasts with the behavior of velocity coefficient where no change in these two zones is found.

The two points with strange behavior of nozzle A (higher back pressure and low Reynolds) are probably wrong due to some error in mass or momentum flux measurement or an accumulation of both ones. 


\section{Analysis}

In the absence of cavitation phenomenon, the value of the area coefficient depends on the velocity profile at the orifice outlet, in such way that $C_{a}$ will be greater the more uniform is the velocity profile. The most important factor that will influence on the profile characteristics is the flow regime. In Figure 15 are presented the velocity profiles for laminar and turbulent regimes.

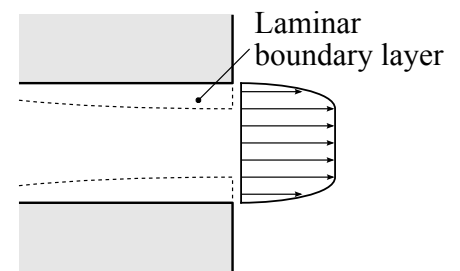

a) Laminar regime

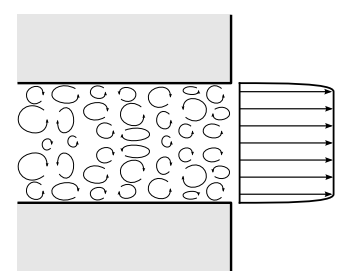

b) Turbulent regime

Figure 15: Comparison between the velocity profiles for laminar and turbulent regimes at the injection orifice outlet.

In laminar regime, since the flow is not fully developed ${ }^{1}$, the profile will depend on the thickness of the boundary layer. In the boundary layer the profile is parabolic while in the central line the velocity is constant. The higher the thickness of this layer is, the less uniform velocity, and therefore the lower $C_{a}$.

On the other hand, in turbulent regime, the velocity profile will be more uniform than in the laminar case, as a result of transverse momentum flux transport caused by turbulence. Consequently, for turbulent regime, $C_{a}$ will be greater than for laminar regime and very close to the unity.

From the two zones of behavior observed in the area coefficient, Figure 14, the zone for high Reynolds numbers agrees strongly with turbulent regime behavior, and therefore, the $C_{a}$ coefficient is very close to the unity.

The other zone, for low $R e$, traditionally has been associated with the laminar regime, for example [17, 20]. However, as it will be shown below, in the low Reynolds number zone, the flow is in the transition zone between laminar and turbulent flow.

Let's annalize first the behavior of laminar regime. The thickness of the laminar boundary layer depends essentially on the fluid velocity and distance

\footnotetext{
${ }^{1}$ In laminar regime in fully developed conditions, in a distance far enough from the orifice inlet, the profile will be completely parabolic.
} 
covered [21], as follows

$$
\delta \propto \sqrt{\frac{\nu x}{u}} .
$$

Thus, the greater velocity the lower will be the thickness of the laminar boundary layer, while the greater is the covered distance, the thickness will be greater. Another factor with influence is the orifice convergence, considering the fluid acceleration when the orifice area decreases, the thickness of the boundary layer decreases due to this higher velocity.

The ratio between the boundary layer thickness and the orifice diameter $\delta / D$, obtained from the equations (20) and taking into account the Reynolds Number, is as follows:

$$
\frac{\delta}{D} \propto \sqrt{\frac{1}{R e} \frac{L}{D}} .
$$

Obviously, considering the velocity profile, the higher this relation, the lower will be $C_{a}$.

Comparing this ratio with the results obtained in the study of diameter influence (Figure 14) is observed that in all nozzles, when the Re increases the $C_{a}$ coefficient increases too (decreases $\delta$ ).

However, it not happens the same with the influence of $L / D$ ratio. From equations (21) the $C_{a}$ coefficient should be greater for the nozzles with the lowest $L / D$. However the results show a completely opposite behavior.

From this analysis it can be concluded that for $R e$ used in this study the nozzles are not completely in laminar regime at any point.

Since laminar flow has been rejected, let's see if the flow can be in transitional regime. In the transition zone between laminar regime and turbulent regime, the flow is characterized by intermittent character. This behavior is characterized by intermittency factor $\gamma$, which defines the fraction of time during which the regime is turbulent in a given point. Thus, $\gamma=1$ would mean a steady turbulent regime and on the opposite side, $\gamma=0$ would mean a fully laminar regime.

Considering the comments about velocity profiles and $C_{a}$ for both regimes, laminar and turbulent, an increase in the intermittency factor would imply more time in turbulent flow, and therefore an increase in the area coefficient.

As demonstrated Rotta experiments [22] (referred in [21]), for inlet duct flow, the intermittency factor depends on the Reynolds number and covered distance in the duct. Thus, $\gamma$ will be greater if $R e$ and/or the covered distance $x / D$ are increased.

Applying this result in the current study, the behavior of $C_{a}$ for all nozzles in the low Reynolds number zone is explained (Figure 14): 
- If the Reynolds number is increased, the intermittency factor is increased in the same proportion. As a consequence, $C_{a}$ also increases.

- If the $L / D$ ratio is increased, there is a higher distance to develop the turbulent flow. This fact will be reflected in an increase in the intermittency factor at the orifice outlet. Again, as a result of this increase the $C_{a}$ also increases. In the Figure 14 it can be seen how $C_{a}$ is higher $L / D$ ratio increases.

- The point where turbulent regime is achieved, which coincides where the maximum $C_{a}$ is reached, occurs at lower $R e$ when the $L / D$ ratio of the injection orifice is increased, since more length is available for turbulence development.

\section{Conclusions}

From the present research work the following conclusions can be stated:

- A study with convergent nozzles with different orifice diameters, and hence different $L / D$ ratio, has been performed. Due to the absence of cavitation phenomenon in the nozzles studied, the effect of turbulence has been specifically studied, otherwise would has been masked.

- It has been found an internal flow behavior differentiated into two zones, which correspond to developed turbulent flow at the orifice outlet, and with the transition zone between laminar and turbulent regimes.

- When injection nozzle flow is in no turbulent regime, discharge coefficient decreases, and spray formation get worse due to the less turbulence. So, attention should be payed for the appearance of this behavior, mainly at low pressure points, by injection nozzle designers or researchers, moreover taking into account that diesel injection nozzles are getting smaller and more convergent.

- Both zones distinction has been realized calculating the area coefficient, since this parameter, in cavitation absence conditions, depends on the velocity profile at orifice outlet, being $C_{a}$ greater as the profile is more uniform. Thus, for the turbulent zone, due to velocity uniform profile is proved $C_{a}$ value is close to the unity. On the other hand, in the transit zone between laminar and turbulent regimes, the $C_{a}$ coefficient is found greater as the intermittency factor on the orifice outlet 
increases, or in another way, the more time spends the flow regime in turbulent conditions, higher is the $C_{a}$ coefficient. The results obtained in this region agree with theory, obtaining a higher $C_{a}$ coefficient if the Reynolds number and/or the $L / D$ ratio are increased, because of the greater intermittency factors in these cases.

- Other effect of the $L / D$ ratio is the increase of wall friction losses when this ratio is increased; this effect is clearly reflected in the results of the velocity coefficient.

\section{Acknowledgements}

This research has been funded in the frame of the Project "Estudio de la influencia del levantamiento de aguja en el proceso de inyección diésel", Reference PAID-06-10-2362, from Universidad Politécnica de Valencia from Spain. The authors thank José Enrique del Rey for his collaboration in the experimental measurements.

\section{References}

[1] Park, S., Kim, J., and Lee, C. Effect of injector type on fuel-air mixture formation of high-speed diesel sprays. Proceedings of the Institution of Mechanical Engineers, Part D: Journal of Automobile Engineering, 220(5):647-659, 2006.

[2] Kastengren, A. and Powell, C. Spray density measurements using x-ray radiography. Proceedings of the Institution of Mechanical Engineers, Part D: Journal of Automobile Engineering, 221(6):653-662, 2007.

[3] Chung, N.H., Oh, B.G., and Sunwoo, M.H. Modelling and injection rate estimation of common-rail injectors for direct-injection diesel engines. Proceedings of the Institution of Mechanical Engineers, Part D: Journal of Automobile Engineering, 222(6):1089-1001, 2008.

[4] Som, S., Aggarwal, S.K., El-Hannouny, E.M., and Longman, D.E. Investigation of nozzle flow and cavitation characteristics in a diesel injector. Journal of Engineering for Gas Turbines and Power, 132(4):042802, 2010 . 
[5] Allocca, L., Andreassi, L., and Ubertini, S. Enhanced splash models for high pressure diesel spray. Journal of Engineering for Gas Turbines and Power, 129(2):609-621, 2007.

[6] Coppo, M. and Dongiovanni, C. Experimental validation of a commonrail injector model in the whole operation field. Journal of Engineering for Gas Turbines and Power, 129(2):596-608, 2007.

[7] Payri, R., Salvador, F., Gimeno, J., and de la Morena, J. Study of cavitation phenomena based on a technique for visualizing bubbles in a liquid pressurized chamber. International Journal of Heat and Fluid Flow, 30(4):768-777, 2009.

[8] Soteriou, C., Zuelch, S., Lambert, M., and Passerel, D. The flow characteristics of high efficiency diesel nozzles with enhanced geometry holes. In THIESEL 2006 Conference on Thermo and Fluid-dynamic Processes in Diesel Engines. 2006.

[9] Payri, R., Garcia, J., Salvador, F., and Gimeno, J. Using spray momentum flux measurements to understand the influence of diesel nozzle geometry on spray characteristics. Fuel, 84(5):551-561, 2005.

[10] Vergnes, C., Foucher, F., and Mounaim-Rousselle, C. Discharge coefficients for a diesel injector during cold starting conditions. Atomization and Sprays, 19(7):621-631, 2009.

[11] Macián, V., Bermudez, V., Payri, R., and Gimeno, J. New technique for determination of internal geometry of a diesel nozzle with the use of silicone methodology. Experimental Techniques, 27(2):39-43, 2003.

[12] Bosch, W. Fuel rate indicator is a new measuring instrument for display of the characteristics of individual injection. SAE Paper 660749, 1966.

[13] Payri, R., Salvador, F., Gimeno, J., and Bracho, G. A new methodology for correcting the signal cumulative phenomenon on injection rate measurements. Experimental Techniques, 32(1):46 - 49, 2008.

[14] Macián, V., Payri, R., Margot, X., and Salvador, F.J. A cfd analysis of the influence of diesel nozzle geometry on the inception of cavitation. Atomization and Sprays, 13:579-604, 2003.

[15] Holman, J. and Gajda, W. Experimental methods for engineers. McGraw-Hill New York, 1994. 
[16] White, F. Fluid mechanics. McGraw-Hill, 2004.

[17] Lichtarowicz, A.K., Duggins, R.K., and Markland, E. Discharge coefficients for incompressible non-cavitating flow through long orifices. $J$. Mech. Engng Sci., 7(2):210-219, 1965.

[18] Fox, T. and Stark, J. Characteristics of miniature short-tube orifice flows. Proc. Inst. Mech Engrs, 203:351-357, 1989.

[19] Fox, T. and Stark, J. Discharge coefficients for miniature fuel injectors. Proc. Inst. Mech Engrs, 203:75-78, 1989.

[20] von Kuensberg Sarre, C., Kong, S.C., and Reitz, R.D. Modeling the effects of injector nozzle geometry on diesel sprays. SAE Paper 199901-0912, 1999.

[21] Schlichting, H. Boundary-Layer Theory. Springer, 2000.

[22] Rotta, J. Experimenteller beitrag zur entstehung turbulenter strömung im rohr. Ingenieur-Archiv, 24(4):258-281, 1956.

Published as: Payri, Raúl; Salvador, F.J. ; Gimeno, Jaime; García Martínez, Antonio. (2012). Flow regime effects over non-cavitating diesel injection nozzles.Proceedings of the Institution of Mechanical Engineers Part D Journal of Automobile Engineering, 226, 1, 133-144. DOI: $10.1177 / 0954407011413056$ 